Mitteilungen der Österreichischen Geographischen Gesellschaft,

155. Jg. (Jahresband), Wien 2013, S. 243-262

\title{
RELIGIONSGEOGRAPHIE
}

\section{Regionale Verteilung von Wallfahrtsorten \\ Choristische Prinzipien der räumlichen Sortierung christlicher Heiligtümer}

\author{
Johannes MAhne-Bieder und Markus HiLPert, beide Augsburg*
}

mit 8 Abb. und 2 Tab. im Text

\section{INHALT}

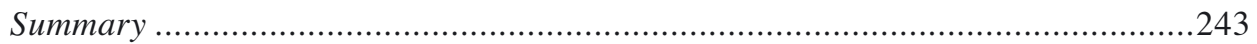

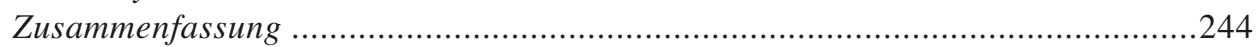

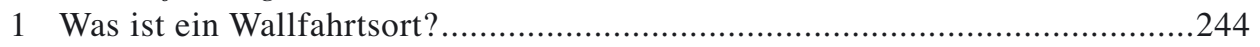

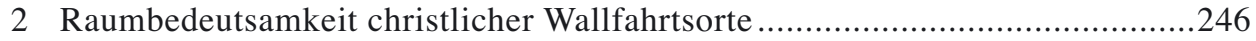

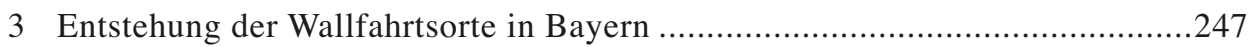

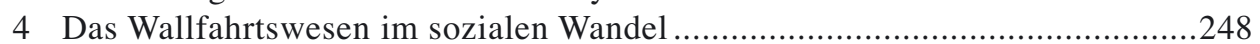

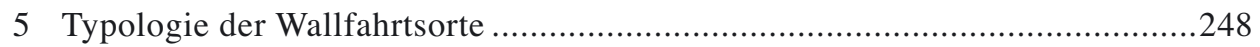

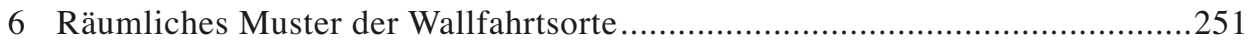

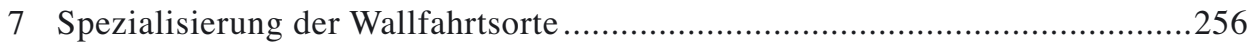

8 Auswirkungen der Spezialisierung auf das räumliche Muster ........................259

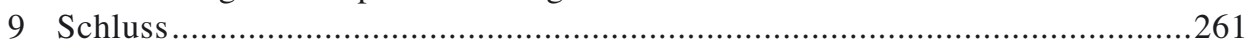

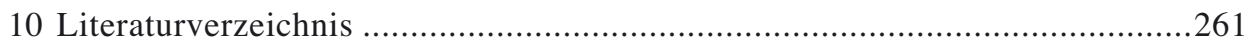

\section{Summary}

Spatial patterns of places of pilgrimage. Choristic principles of sorting Christian sanctuaries

Places of pilgrimage are places for the special worshipping of religious cult artifacts. In particular during the Middle Ages, a close-meshed network of sanctuaries

\footnotetext{
* Dipl.-Geogr. Johannes Mahne-Bieder und PD Dr. Markus Hilpert, beide Lehrstuhl für Humangeographie und Geoinformatik, Institut für Geographie, Universität Augsburg, Universitätsstraße 10, D-86159 Augsburg, Deutschland; E-Mail: johannes.mahne@geo.uni-augsburg.de; markus.hilpert@geo. uni-augsburg.de, http://www.geo.uni-augsburg.de
} 
emerged, which has in fact largely remained unchanged until today. Nevertheless, based on their liturgical and secular destinations it is today possible to statistically identify different types of sanctuaries and bring them into a hierarchical order similar to the theory of Central Places of Walter CHRISTALLER. Similarly, recurrent spatial patterns can be discerned. In correspondence with that, it is possible to identify differences in demand that, in turn, correspond statistically with different types of visitors. Fluctuations in demand partly result in a specialisation of sanctuaries on the same hierarchy level, just as formulated by Alfred LösCH in his theory of market networks. Such spatial results of specialisation processes are particularly visible in the size of the catchment areas.

\section{Zusammenfassung}

Wallfahrtsstätten sind Orte besonderer Verehrung von religiösen Kultobjekten (Gnadenbild, Heiligengrab, Reliquie etc.). Vor allem im Mittelalter entwickelte sich ein sehr engmaschiges Netzwerk von Wallfahrtsorten, das im Grunde bis heute kaum eine Veränderung erfuhr. Allerdings können heute anhand des liturgischen und weltlichen Angebots unterschiedliche Typen von Wallfahrtsstätten statistisch differenziert und in eine hierarchische Ordnung gebracht werden, die jener der Zentralen-Orte-Theorie nach Walter CHRISTALLER ähnelt. Gleichsam lassen sich regelmäßige räumliche Muster nachweisen. Damit korrespondierend sind Unterschiede in der Nachfrage festzustellen, die statistisch mit verschiedenen Besuchertypen korrespondieren. Die variable Nachfrage generiert teilweise auch eine Spezialisierung von Wallfahrtsstätten derselben Hierarchiestufe, wie sie Alfred LösCH in der Theorie der Marktnetze formuliert hat. Besonders an der Größe der Einzugsgebiete hochspezialisierter Wallfahrtsorte lassen sich solche räumliche Auswirkungen der Spezialisierung erkennen.

\section{Was ist ein Wallfahrtsort?}

Wallfahrtsstätten in der christlichen Religion sind Orte der besonderen Verehrung von Kultobjekten (Gnadenbild, Heiligengrab, Reliquie etc.) mit einer überörtlichen Raumbedeutsamkeit (Zentralität). Bevor sich allerdings die Raumwissenschaften der Untersuchung von Wallfahrtsstätten zuwandten, hat vor allem die Volkskunde das Wallfahrtswesen analysiert und definiert. Demnach ist jeder Ort, der über ein verehrtes Kultobjekt verfügt, als Wallfahrtsort zu bezeichnen (KRISS 1956, S. 26f.). Da sich die Verehrung zumeist auch materiell manifestiert, galt folglich das Forschungsinteresse vor allem den Votivgaben der Gläubigen. ${ }^{1)}$

\footnotetext{
1) Votivgaben werden zum Dank (aufgrund einer Gebetserhörung oder der Rettung aus einer Notlage) von Gläubigen an einem Heiligtum hinterlassen. Es können Votivtafeln (bildliche Darstellungen), Wachsvotive und Metallvotive (meist aus Silber), welche oft auch die erkrankten Körperteile nachbilden, unterschieden werden.
} 
In jüngerer Vergangenheit setzte sich auch eine alternative Wallfahrt-Definition in der Volkskunde durch, die in erster Linie auf dem prozessionsartigen Aufsuchen eines Ortes durch Gläubige basiert. So sei nur dort ein Wallfahrtsort existent, wo Wallfahrten - und zwar in Gruppen und prozessionsartig - zu diesem Ort stattfinden (vgl. BRÜCKNER 2000).

Interessanterweise vermeidet das kanonische Recht der Katholischen Kirche den Begriff Wallfahrtsort und spricht stattdessen von einem Heiligtum, das ,aus besonderem Frömmigkeitsgrund [von] zahlreichen Gläubigen mit Gutheißung des Ortsordinarius“ aufgesucht wird. Die kirchenrechtliche Anerkennung als Heiligtum (Wallfahrtsort) erfolgt dann ex-post (Ioannes Paulus PP. II \& Aymans 2001, S. 537).

Gleichermaßen entbrannte um den Begriff Wallfahrer eine langjährige Diskussion in der Volkskunde. So definierte KRIss jeden Gläubigen, der dem „Bedürfnis des menschlichen Herzens, der Gottheit von Zeit zu Zeit besonders nahe zu kommen“, nachkommt, indem er eine religiös motivierte Reise zu einem besonders verehrten Ort unternimmt, als Wallfahrer (1956, S. 26). KRIss prägte so den Begriff des Einzelwallfahrers. Diese Auffassung steht im Gegensatz zur Definition BRÜCKNERs.

Diese Unterschiede in den Definitionen von Wallfahrt und Wallfahrern resultieren aus verschiedenen religionsgeographischen Grundannahmen. KRIss vertritt die Auffassung, dass Definitionen von Wallfahrt und Wallfahrer interreligiös und international anwendbar sein müssen, da außer dem Christentum alle großen Weltreligionen entsprechende Phänomene kennen, die als Wallfahrt bezeichnet werden können (1963, S. 101-107). Für BRÜCKNER hingegen ist der Begriff Wallfahrt und auch der des Wallfahrers nur auf bestimmte Formen des religiösen Reisens anwendbar, nämlich das Aufsuchen eines Heiligtums in der Gruppe und in der Form einer Prozession (2000, S. 233-251).

Der vorliegende Aufsatz folgt bei der Verwendung des Begriffs Wallfahrtsort der Definition des kanonischen Rechts. Es erscheint beispielsweise nicht angemessen, weltlich und kirchenrechtlich anerkannte Heiligtümer nicht als Wallfahrtsorte anzusprechen, wenn diese zwar aktuell nicht mehr das Ziel von prozessionsartigen Wallfahrerzügen sind, jedoch noch von Einzelpersonen in religiösen Anliegen aufgesucht werden. Ebenso werden auch Einzelpersonen als Wallfahrer definiert, sofern sie aus religiösen Motiven den Wallfahrtsort aufsuchen. Denn heute hat die persönliche religiöse Praxis (im Christentum vor allem das Gebet) einen zumindest gleich bedeutsamen Stellenwert wie die öffentliche religiöse Praxis (z.B. der Gottesdienstbesuch), zu der auch die Unternehmung einer prozessionsartigen Wallfahrt in einer Gruppe zu zählen ist. So zeigen etwa bei der demoskopischen Untersuchung des Religionsmonitors 2008 lediglich 27\% der befragten Katholiken eine hohe Intensität bei der öffentlichen religiösen Praxis.$^{2)}$ Bei der privaten religiösen Praxis ${ }^{3)}$ steigt der Anteil der Gläubigen mit einer hohen Intensität indes auf 42\% (HuBER 2008b, S. 14ff.).

2) Öffentliche religiöse Praxis meint die Teilnahme an öffentlichen religiösen Ritualen. Im Religionsmonitor 2008 wird die Intensität mithilfe der Frage nach der Häufigkeit der Teilnahme an solchen Ritualen und der beigemessenen Bedeutung ermittelt (HuBER 2008b, S. 13-15).

3) Private religiöse Praxis meint die Hinwendung zur Transzendenz außerhalb der öffentlichen Rituale. Im Christentum ist hier vor allem das Gebet gemeint. Die Intensität wird analog zur öffentlichen religiösen Praxis gemessen (Huber 2008b, S. 15-18). 
Eine hohe Dichte und eine große Anzahl an Wallfahrtsorten findet sich im Bistum Augsburg, das räumlich fast identisch mit dem Regierungsbezirk Bayerisch-Schwaben ist. Vor allem Mittelschwaben ist gekennzeichnet durch einen besonderen Reichtum entsprechender Elemente der sakralen Kulturlandschaft. Am Beispiel dieser Untersuchungsregion widmet sich der vorliegende Aufsatz der Suche nach räumlichen Verteilungsmustern dieser Gnadenorte. Dazu dienen auch eine Typisierung der Wallfahrtsorte sowie die Klassifizierung ihrer Besucher mittels multivariater Statistik. $\left.{ }^{4}\right)$

\section{Raumbedeutsamkeit christlicher Wallfahrtsorte}

Im Christentum hat die Kirche als Gebäude eine herausragende Bedeutung für die Glaubenspraxis. Die praktische Ausübung des Glaubens ist stark an die Kirche als Ort des Gottesdienstes und des Gebets gebunden. Kirchen und andere sakrale Gebäude haben daher im christlichen Europa eine raumprägende Wirkung. Gerade in ländlichen Räumen sind sie zumeist die höchsten Gebäude einer Siedlung. Dieser architektonische Aspekt unterstreicht ihren besonderen Stellenwert. Sie sind häufig die am weitesten im Umland sichtbaren Ausdrücke menschlichen Bauens in der heutigen Kulturlandschaft (Rinschede 1999, S. 148f.).

Aber auch andere sakrale Einrichtungen haben den Raum geprägt. So ist die Urbarmachung großer Gebiete in Deutschland (Innere Kolonisation) auf die Anwesenheit von Klöstern zurückzuführen. Viele Orden siedelten sich in wenig erschlossenen Gebieten an und formten die Naturlandschaft zur heutigen Kulturlandschaft um. So bevorzugte der Benediktiner-Orden beispielsweise Lagen auf Anhöhen oder Bergen, der Zisterzienser-Orden hingegen siedelte vornehmlich in peripheren Tälern. Darüber hinaus gibt es zahlreiche Rodungsklöster, die die Kulturlandschaft in bisher unerschlossenes Waldgebiet ausdehnten (RINSCHEDE 1999, S. 152f.).

Das frühe Christentum entwickelte rasch einen Märtyrerkult, welcher im Laufe der Jahre zu einem Heiligenkult transformierte. Nach dem Ende der Christenverfolgung im Römischen Reich entstanden dadurch die ersten Grabes- und Gedenkkirchen. Diese dienten der Verehrung der Märtyrer und wurden früh zu Zielen religiöser Reisen der Gläubigen. Die Märtyrergräber in Rom [Roma] - die heute noch von zahlreichen Gläubigen besucht werden - sind deshalb die ältesten Wallfahrtsorte Europas (RINSCHEDE 1999, S. 148f.). Prälat IMKAMP, der Wallfahrtsdirektor von Maria Vesperbild in Schwaben, bezeichnete in einem Interview die Wallfahrt sogar als die ,älteste Form des Massentourismus“ (IMKAmp zitiert nach Renkikowski 2009,). Anders formuliert sind religiös motivierte Reisen , die geographisch signifikantesten Formen [...] religiösen Verhaltens“ (RINSCHEDE 1999, S. 197). Seine Raumbedeutsamkeit generiert der Religionstourismus aber vor allem durch entsprechende Infrastrukturen. Hierzu zählen beispielsweise neben Wegen (z.B. Jakobuspilgerweg) sowie Einkehr- und Versorgungsmöglichkeiten in erster Linie die Zielpunkte der Reise, die Wallfahrtsorte.

\footnotetext{
4) Der Aufsatz basiert auf einer nicht veröffentlichten Arbeit von Johannes MAHNE-BIEder. Wenn nicht
} anders angegeben, basieren die Aussagen auf dieser Arbeit. 
Ein von der Religionsgeographie - im Hinblick auf seine Raumbedeutsamkeit besonders intensiv erforschter Gnadenort ist Lourdes in Frankreich. Vor der berichteten Marienerscheinung eines Mädchens im Jahr 1858, auf der die Wallfahrt beruht, war Lourdes eine kleine, von der Landwirtschaft geprägte Siedlung. Bis heute hat sich Lourdes aber in Folge der zahlreichen Religionstouristen (ca. 4,5 Mio. Besucher pro Jahr) stark gewandelt. So besitzt der Marienwallfahrtsort seit 1948 sogar einen eigenen Flughafen (RINSCHEDE 1985, S. 218). Annähernd die gesamte wirtschaftliche Leistung des Ortes basiert zudem auf der Wallfahrt.

Andere Wallfahrtsorte sind religionsgeographisch bisher zwar nicht annähernd in gleichem Umfang untersucht worden. Dennoch gibt es auch in Deutschland Orte, die maßgeblich von ihren Heiligtümern wirtschaftlich profitieren, beispielsweise Altötting in Bayern. Gleichwohl ist die sakrale Kulturlandschaft im bayerisch-schwäbischen Raum im Allgemeinen und die Geographie der Wallfahrtsorte im Besonderen kaum durch einzelne herausragende Gnadenorte, sondern mehr durch ein besonders reichhaltiges Angebot charakterisiert. Gerade im Bistum Augsburg ist die Dichte der Wallfahrtsorte besonders hoch.

\section{Entstehung der Wallfahrtsorte in Bayern}

Die ersten bayerischen Wallfahrtsorte entstanden im 8. Jh. durch die Verehrung von Reliquien. Seit ottonischer Zeit (ab 947 n. Chr.) wurden die Reliquien in mehrjährigem Turnus bei sogenannten Heiltumsweisungen dem Volk gezeigt. Dabei wurden auch Ablässe (Nachlass der zeitlichen Sündenstrafen) gewährt, die bald zum Hauptgrund vieler Wallfahrten wurden. Da es aber nur wenige Orte mit Heiltumsweisungen gab, mussten die Gläubigen oft weite Reisen auf sich nehmen (HöLlhuber \& Kaul 1987, S. 52-55).

Nach den Kreuzzügen, zu Beginn des 13. Jhs., war eine Fülle verschiedener Reliquien in ganz Europa verbreitet. Gleichzeitig florierte noch immer das Ablasswesen. So nahm die Dichte der Kultobjekte und der Orte mit der Möglichkeit der Erlangung eines Ablasses stark zu. Zur gleichen Zeit setzte die Verehrung des „wahren Kreuzes“ ein und die Wallfahrten zu Kreuzpartikeln breiteten sich rasch aus. Sprunghaft stieg zudem die Zahl der Wallfahrtsorte mit der Einführung des Gnadenbildes im Hochmittelalter an. Durch die gestiegene Anzahl an Kultobjekten und das Bedürfnis der fußläufigen Erreichbarkeit entstand so eine Vielzahl an Nahwallfahrten in ehemals kleineren Kultorten (Höllhuber \& Kaul 1987, S. 55-62).

Seit dieser Zeit hat sich die Anzahl der Wallfahrtsorte kaum noch verändert. Im Bistum Augsburg gibt es heute 227 Heiligtümer und zwei Gebetsstätten (von der Katholischen Kirche nicht offiziell anerkannte Heiligtümer) (Bistum Augsburg 2011). Die Gebetsstätten (Marienfried und Wigratzbad) stellen in zweierlei Hinsicht eine Besonderheit dar. Beide haben ihren Ursprung im Widerstand gegen das Dritte Reich und sind somit vergleichsweise junge Orte des Glaubens. Sie heben sich jedoch auch im Angebot von den anderen Wallfahrtstätten ab: Beide Gebetsstätten bieten den Gläubigen vorkonsiliarische Formen der Frömmigkeit (z.B. Zelebration der Messe in 
Latein). Bei Betrachtung der Lage ist augenfällig, dass beide Stätten an Lücken im Netz der religiösen Oberzentren entstanden sind (vgl. Abb. 2 und 3).

\section{Das Wallfahrtswesen im sozialen Wandel}

Die gesellschaftlichen Transformationen in der zweiten Hälfte des 20. Jhs. hatten verschiedene Auswirkungen auf das Wallfahrtswesen: Durch die Massenmotorisierung seit den 1950er und 1960er Jahren verlor die fußläufige Erreichbarkeit der Wallfahrtsorte an Bedeutung und die soziale Säkularisierung in den 1970er und 1980er Jahren führte zu einem weiteren Bedeutungsverlust vieler Nahwallfahrten und vor allem des Ablasswesens. Hinzu kam eine Veränderung des Freizeitverhaltens, sodass beispielsweise auch Argumente des Fremdenverkehrs oder der Naherholung das Wallfahren beeinflussen (Höllhuber \& Kaul 1987, S. 84-90).

In den letzten Jahren lässt sich jedoch wieder ein zunehmendes Interesse an Spiritualität feststellen (RIEGER 2007, S. 11). Dies hat direkte Auswirkungen auf das Wallfahrts- und Pilgerwesen. Nicht zuletzt der Bestseller ,Ich bin dann mal weg“ des deutschen Fernsehmoderators Hape Kerkeling ist Ausdruck dieses neuen Trends. Allerdings ist eine simple Rückkehr zur früher geübten Praxis des Wallfahrens unwahrscheinlich. Zu beobachten sind vielmehr erste Anzeichen einer Neuorganisation des Angebots und der Nachfrage. Wallfahrten dienen heute nicht nur der Bitte, Buße oder dem Dank, sondern vermehrt der spirituellen Selbstfindung oder der sportlichen Aktivität - auch für Nicht-Christen. Dementsprechend reagieren auch die Wallfahrtsorte mit einer Anpassung in Angebot und Ausstattung.

\section{Typologie der Wallfahrtsorte}

Um unterschiedliche Typen von Wallfahrtsorten zu identifizieren, wurde auf Basis einer empirischen Erhebung aller Wallfahrtsorte im Bistum Augsburg durch das Referat für Glaubenslehre der entsprechenden Diözese eine Clusteranalyse durchgeführt. Dabei dienten nicht die Besucherzahlen der Gnadenstätten als distinguierendes Merkmal für die Typisierung, sondern das liturgische Angebot: Erstens spiegelt ein breites und großes Angebot die Zentralität der Wallfahrt wieder, zweitens lassen sich daraus auch Rückschlüsse auf die Menge der religiösen Besucher ziehen. ${ }^{5)}$ Ein

\footnotetext{
5) Beispielsweise liegen kaum belastbare Datenmassive über Besucherzahlen vor, weil nur an zwei Tagen im Jahr (zweiter Fastensonntag und zweiter Sonntag im November) die Besucher der Gottesdienste gezählt werden. Da aufgrund der weitestgehend willkürlichen Terminsetzung der beiden Erhebungen starke Verzerrungen der Ergebnisse zu erwarten sind, kann dieses Merkmal nicht zur Klassifizierung dienen. Darüber hinaus eignet sich die Besucherzahl auch nur sehr eingeschränkt als Indikator für die religiöse Zentralität eines Wallfahrtsortes, weil beispielsweise nicht alle Besucher eines Wallfahrtsortes diesen aus religiösen Motiven aufsuchen. So besichtigt etwa ein Großteil, vor allem der ausländischen Besucher die Wieskirche in Oberbayern aus touristischen Motiven.
} 


\begin{tabular}{|c|c|c|c|c|c|}
\hline \multirow{2}{*}{$\begin{array}{l}\text { Abgrenzungs- } \\
\text { merkmale }\end{array}$} & \multicolumn{5}{|c|}{ Typen der Wallfahrtsorte } \\
\hline & $\begin{array}{c}\text { Religiöse } \\
\text { Oberzentren }\end{array}$ & $\begin{array}{c}\text { Religiöse } \\
\text { Mittelzentren }\end{array}$ & $\begin{array}{c}\text { Mögliche } \\
\text { religiöse } \\
\text { Mittelzentren }\end{array}$ & $\begin{array}{c}\text { Religiöse } \\
\text { Unterzentren }\end{array}$ & $\begin{array}{c}\text { Religiöse } \\
\text { Kleinzentren }\end{array}$ \\
\hline Gottesdienst & ++ & ++ & + & -- & + \\
\hline Beichtangebot & ++ & + & + & -- & + \\
\hline $\begin{array}{l}\text { liturgisches } \\
\text { Angebot }\end{array}$ & ++ & + & - & -- & - \\
\hline Feste & ++ & + & - & - & -- \\
\hline Exerzitien & ++ & + & -- & -- & -- \\
\hline Außenanlagen & ++ & + & - & -- & -- \\
\hline Pilgerladen & ++ & -- & -- & -- & -- \\
\hline Schriftenstand & ++ & + & - & - & - \\
\hline $\begin{array}{l}\text { Übernachtungs- } \\
\text { möglichkeiten }\end{array}$ & ++ & -- & -- & -- & -- \\
\hline Werbung & ++ & + & -- & -- & -- \\
\hline
\end{tabular}

Tab. 1: Abgrenzungskriterien der Typen von Wallfahrtsorten

vielfältiges liturgisches Angebot würde bei einer mangelnden Nachfrage durch die Gläubigen nicht weiter aufrechterhalten werden. Daher stellt das liturgische Angebot eines Wallfahrtsortes einen belastbaren Indikator für die religiöse Zentralität und die Nachfrage des Gnadenortes durch die Gläubigen dar.

Durch verschiedene Merkmalsausprägungen des liturgischen Angebots der Heiligtümer konnten mittels der Clusteranalyse fünf unterschiedliche Typen identifiziert werden: religiöse Oberzentren, religiöse Mittelzentren, mögliche religiöse Mittelzentren, religiöse Unterzentren und religiöse Kleinzentren (vgl. Abb. 1). Die Typisierung

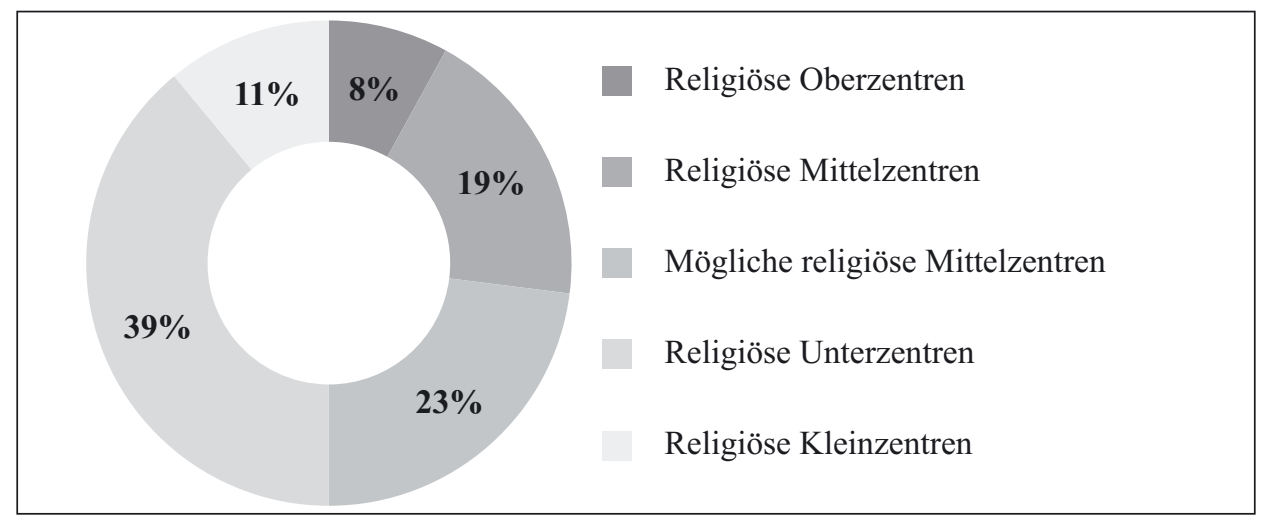

Abb. 1: Typen von Wallfahrtsorten 
weist nicht nur in ihrer Nomenklatur deutliche Gemeinsamkeiten mit der ZentraleOrte-Theorie und den Fachtermini der Raumordnung auf. Auch die Methode der Klassifizierung ist der raumplanerischen Praxis sehr ähnlich und folgt zu Teilen der Katalogmethode.

Durch die Clusteranalyse konnten die Angebote der Wallfahrtsorte in ihrer Breite und ihrer Häufigkeit für jeden einzelnen Typ definiert werden. So ist es ex post möglich, bei Veränderungen im Angebot oder bei Synopsen mit anderen Räumen ohne eine erneute Clusteranalyse einen Wallfahrtsort eindeutig einer Kategorie zuzuordnen.

„Religiöse Oberzentren“ (8\%) verfügen über ein breites Angebot an Gottesdiensten, die zumeist täglich stattfinden. Ebenso häufig kann die Beichte abgelegt werden. Diese Wallfahrtsorte haben folglich das reichhaltigste Angebot an Festen und Feierlichkeiten. Als besondere Alleinstellungsmerkmale sind oft eigene Übernachtungsmöglichkeiten, die Ausstattung mit Geschäften (mit einem speziell auf die Besucher ausgerichtetem Sortiment) und die Bewerbung der Wallfahrtsstätte prägend.

Die ,religiösen Mittelzentren“ (19\%) haben ein ähnlich breites Angebot an Gottesdiensten, jedoch werden zumeist weniger Kirchenfeste im Jahresverlauf entsprechend aufwändig gefeiert. Auch gibt es in der Regel keinen Wallfahrtsladen und auch keine, von der Wallfahrtsstätte selbst getragenen Beherbergungsbetriebe.

Die „möglichen religiösen Mittelzentren“ (23\%) zeichnen sich durch ein noch geringeres Angebot an Festen und Feierlichkeiten im Jahresverlauf aus. Die Wallfahrtsorte dieses Typs genießen daher nur örtliche Bekanntheit. Bei einer geringfügigen Ausweitung des Angebots können diese Orte aber die Rolle „religiöser Mittelzentren“ einnehmen (daher die Bezeichnung „mögliche religiöse Mittelzentren“).

In den „religiösen Unterzentren“ (39\%) werden nur selten Kirchenfeste gefeiert und auch das Angebot an Gottesdiensten ist gering. Dies lässt sich zum Teil mit ihrem kirchenrechtlichen Status erklären. Ein Großteil der Orte dieses Typs sind Kapellen oder Filialen einer Pfarrkirche. Kapellen werden normalerweise nicht für religiöse Rituale genutzt, Filialen nur dann, wenn beispielsweise die Pfarrkirche der Gemeinde nicht ausreichend groß ist oder die Pfarrgemeinde ein großes Gebiet umfasst. Es ist daher eher verwunderlich, dass hier überhaupt liturgische Angebote zu finden sind. Dies lässt sich auf die Wallfahrtsfunktion dieser Orte zurückführen. Zumeist sind die wenigen liturgischen Angebote auf einen kurzen Zeitraum, zum Teil nur einen einzigen Tag im Jahr, beschränkt. In der restlichen Zeit ruhen diese Orte und dienen nur einzelnen Besuchern als Stätte des Gebets.

Die unterste Stufe des hierarchischen Systems der Wallfahrtsorte bilden die ,religiösen Kleinzentren“ (11\%). Hier ist die Wallfahrtsfunktion sehr eingeschränkt, das Angebot an Gottesdiensten ist jedoch höher als bei den „religiösen Unterzentren“, weil viele der „religiösen Kleinzentren“ auch die Funktion einer Pfarrkirche ausüben. 


\section{Räumliches Muster der Wallfahrtsorte}

Die Gnadenorte des Bistums Augsburg konnten aufgrund ihres Angebots an unterschiedlichen Heilsmitteln und Ausstattungsmerkmalen in eine hierarchische Ordnung gebracht werden, die nicht nur sprachlich mit der Theorie der Zentralen Orte nach Walter Christaller (1933) korrespondiert. Bereits Rinschede apostrophiert ein „Netz von Pilgerzentren“ sowie ein „System religiöser Oberzentren“, in das ,ein Netz untergeordneter regionaler und lokaler Stätten“ eingebunden ist (1999, S. 207). RINSCHEDE verzichtet jedoch auf eine Begründung seiner Wortwahl ebenso wie auf eine Beschreibung des Systems.

Eine Anwendung der Zentrale-Orte-Theorie auf Wallfahrtsorte ist sinnvoll, wenn die liturgischen Heilsmittel als religiöse Dienstleistungen des Heiligtums interpretiert werden. Folglich nimmt die Zentralität eines Wallfahrtsorts mit seinem sakralen Angebot zu. Die hierarchische Ordnung der Gnadenstätten basiert gleichsam auf den Angeboten, sodass die Voraussetzung für die Untersuchung räumlicher Muster gegeben ist. Die Reichweiten der einzelnen religiösen Güter können nicht exakt ermittelt werden, da diese noch durch Spezialisierungsprozesse überformt werden (vgl. Kap. 8). Jedoch ist davon auszugehen, dass der Gläubige gewillt ist, für die sporadische Teilnahme an Wallfahrtsfesten längere Wege zurückzulegen, als für die wöchentliche Gottesdienstteilnahme.

Bereits bei der Analyse der Wallfahrtsorte höchster Zentralitätsstufe (vgl. Abb. 2) fallen erste Regelmäßigkeiten auf: Sie unterliegen keinen Ballungstendenzen, sondern sind relativ gleichmäßig über das Untersuchungsgebiet verteilt. Mehr noch: Wird ein regelmäßiges Netz aus Hexagonen über die Karte gelegt, zeigen sich verblüffende räumliche Muster, wie etwa das - freilich abhängig von der (historischen) Bevölkerungsdichte - relativ regelmäßige Netzwerk (vgl. Abb. 2). Zur Abstraktion dieser Befunde muss aus der realen Lage der Gnadenstätten höchster Stufe eine idealtypische Verteilung abgeleitet werden (vgl. Abb. 3). Die bestehenden Lücken im Netz der ,religiösen Oberzentren“ schließen sich gleichsam durch Gnadenstätten außerhalb des Bistums Augsburg, die eine ähnliche Bedeutung haben.

Zusätzlich zur regelmäßigen Verteilung der Gnadenorte höchster Zentralitätsstufe ergeben sich auch Zusammenhänge zwischen Gnadenstätten unterschiedlicher Zentralitätsstufen. Besonders deutlich lässt sich dies am Beispiel des sogenannten Heiligen Bergs bei Andechs erläutern (vgl. Abb. 4). Dieser Wallfahrtsort gehört zu den ,religiösen Oberzentren“, ihn umgeben ringförmig Gnadenorte der zweiten und dritten Hierarchiestufe. Da sich diese kreisförmigen Einzugsgebiete der Gnadenorte der unterschiedlichen Hierarchiestufen überschneiden, lassen sich, um eine flächendeckende Versorgung ohne Lücken oder Überschneidungen zu gewährleisten, die „Marktgebiete“ in Hexagone formen und die reale Lage in eine idealisierte Form transformieren (vgl. Abb. 5). 


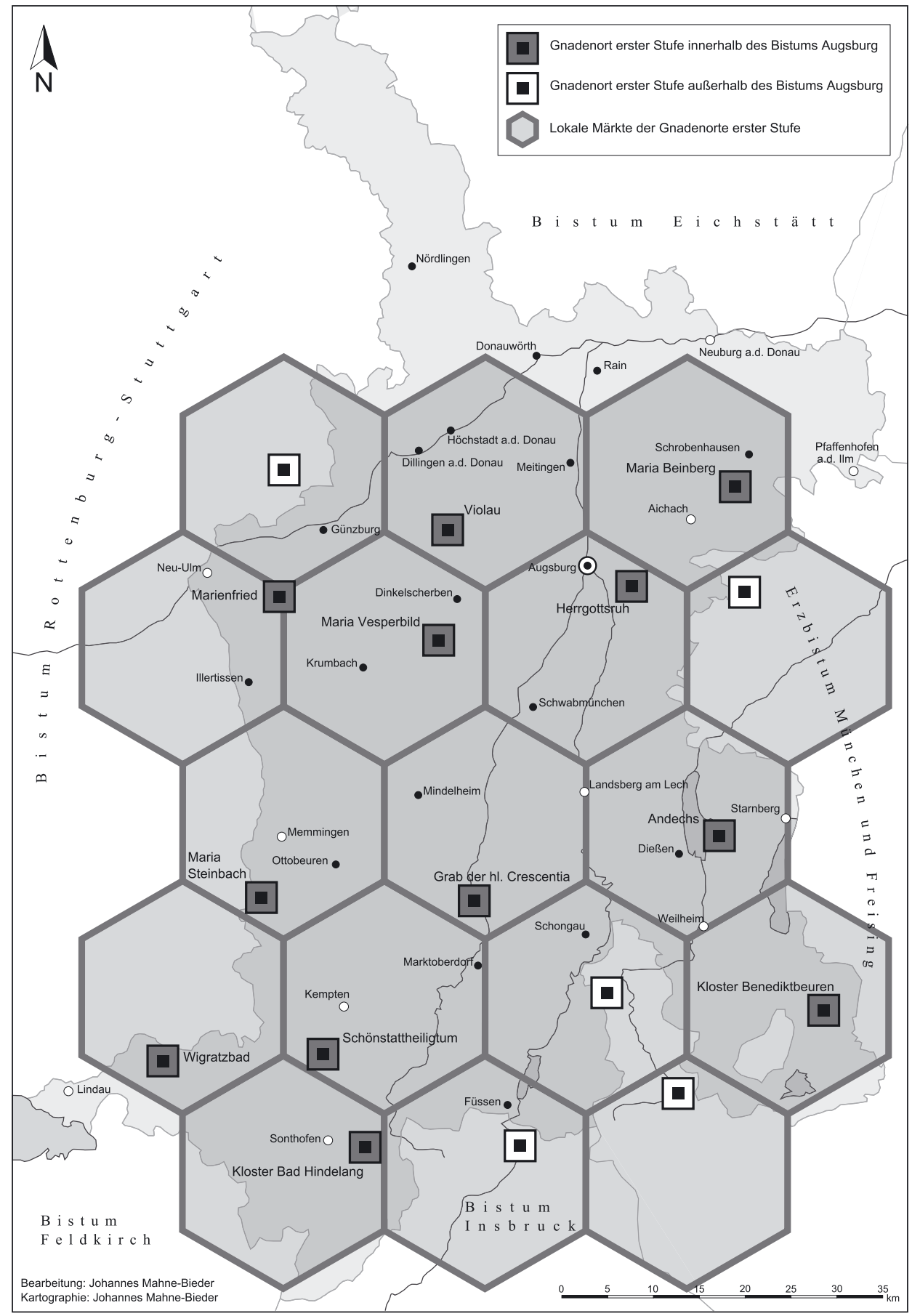

Abb. 2: Reale Lage der religiösen Oberzentren 


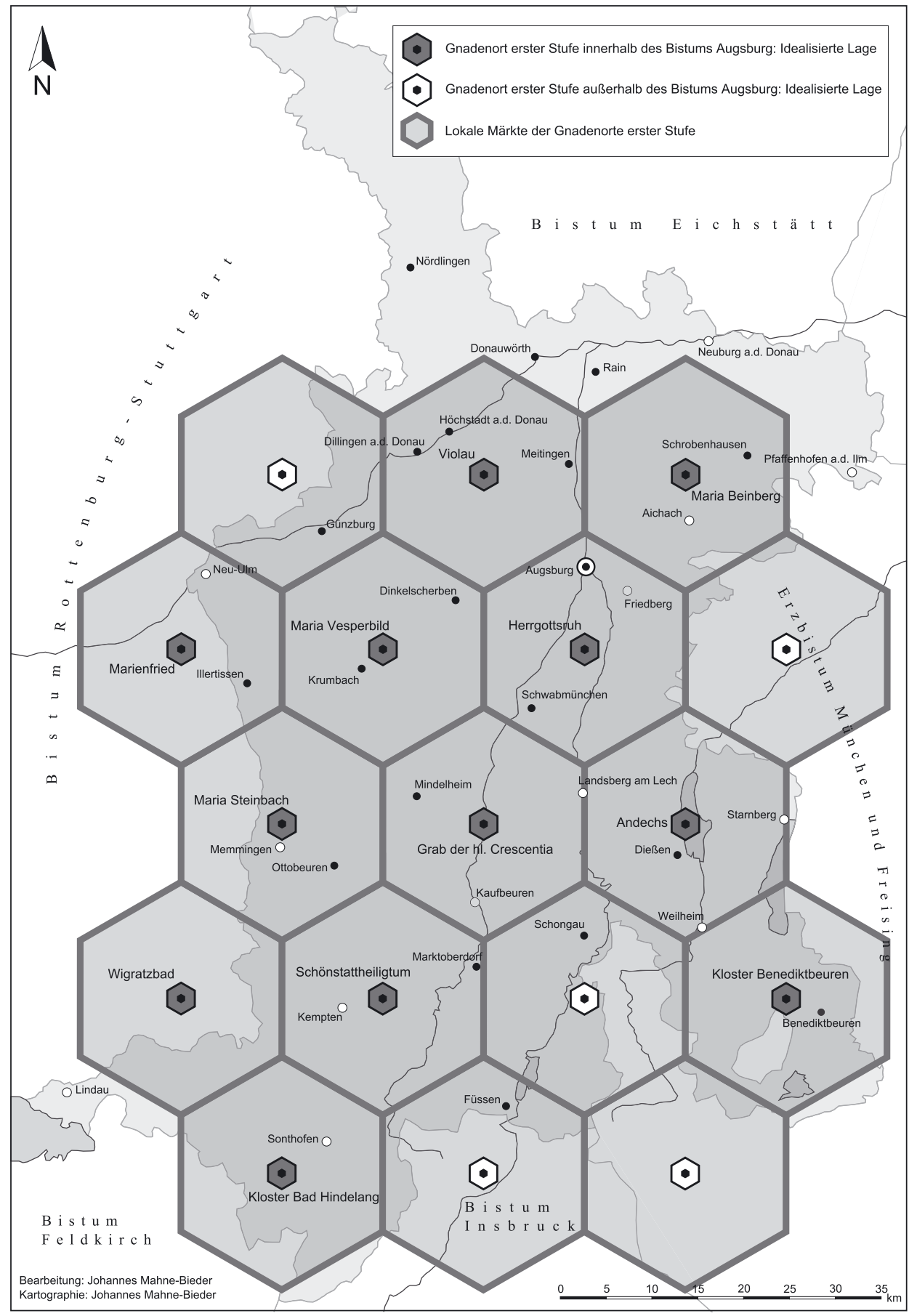

Abb. 3: Idealisierte Lage der religiösen Oberzentren 


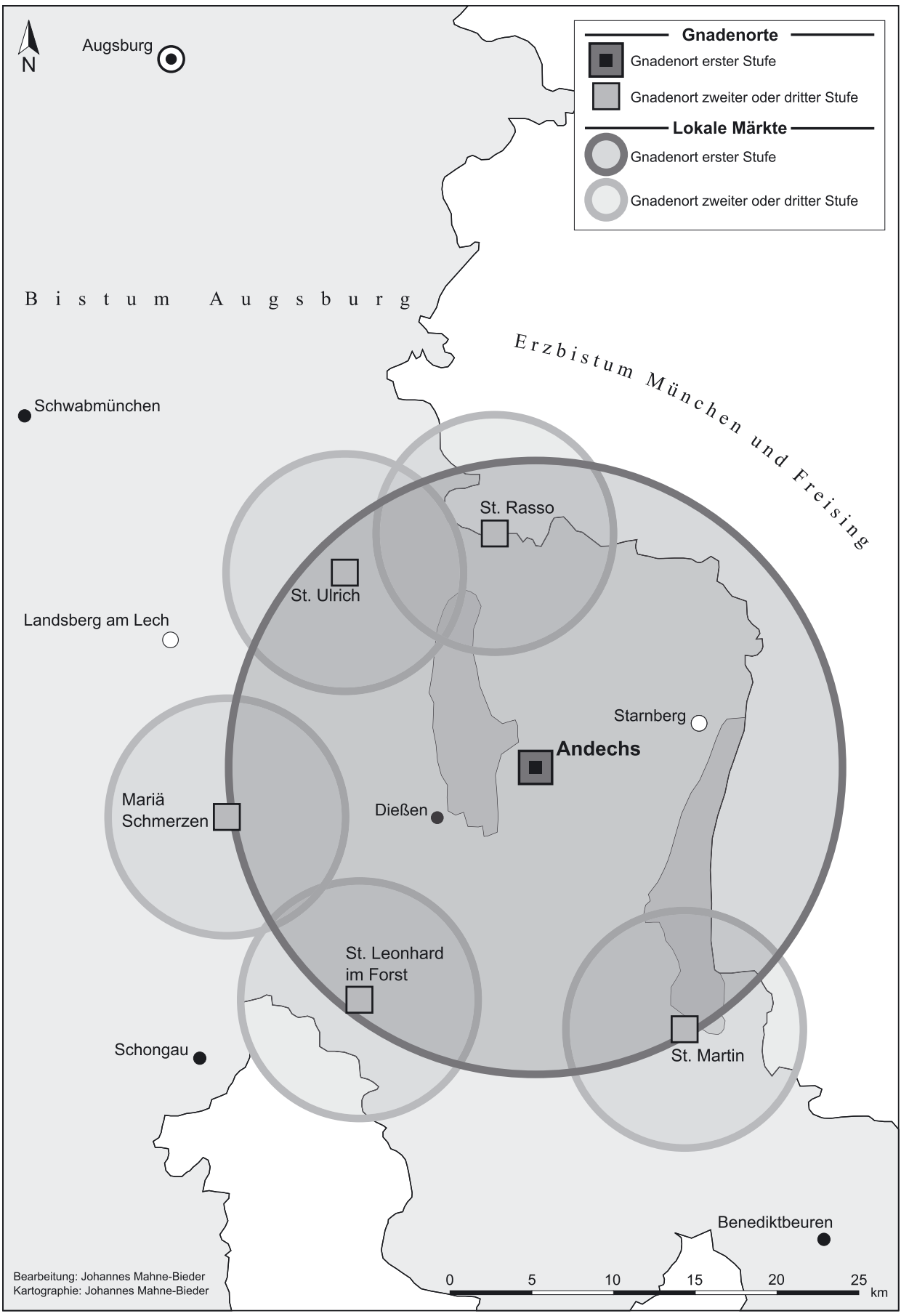

Abb. 4: Kreisförmige Marktgebiete 


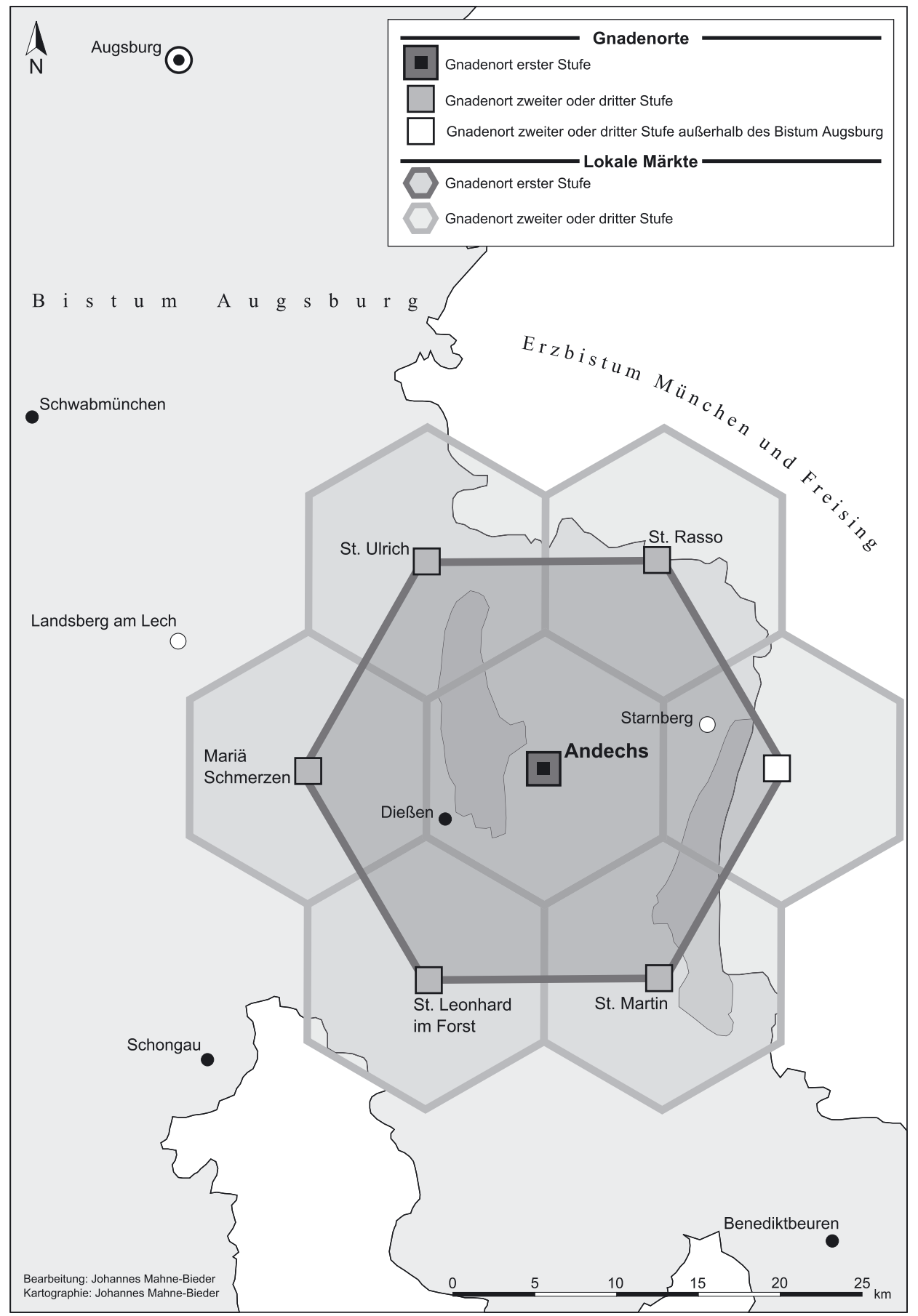

Abb. 5: Idealisierte Marktgebiete 


\section{Spezialisierung der Wallfahrtsorte}

Trotz eindeutiger (clusterbasierter) Kategorien unterscheiden sich Wallfahrtsorte hinsichtlich ihrer Nachfrage und ihres Angebots deutlich voneinander. Die zwei entscheidenden Faktoren bei der Spezialisierung der Wallfahrtsstätten sind das spezifische liturgische und weltliche Angebot sowie die unterschiedlichen Besuchertypen (Nachfrager mit verschiedenen Ansprüchen).

Um unterschiedliche Besuchertypen identifizieren zu können, wurden an ausgewählten Gnadenorten demoskopische Befragungen durchgeführt. Die Besucher wurden dabei zunächst nach den Motiven ihres Besuchs des Wallfahrtsortes gefragt. Hieraus lässt sich eine Unterteilung in touristisch oder religiös geprägte Motivationen ableiten. Neben einigen sozio-demographischen Fragen wurden die Besucher zudem nach der Häufigkeit ihrer Besuche von Wallfahrtsorten interviewt. Abschließend wurde noch die Religiosität der Befragten erfasst. Dabei wurde die Methodik des Religionsmonitors 2008 verwendet. $\left.{ }^{6}\right)$ Die entsprechenden Fragen wurden allerdings so modifiziert, dass sie ausschließlich für Befragte christlicher Konfessionen zutreffen.

\begin{tabular}{|l|l|}
\hline Kerndimensionen & Messung der Intensität \\
\hline Intellekt & Wie oft denken Sie über religiöse Themen nach? \\
\hline Ideologie & Wie stark glauben Sie daran, dass es Gott oder etwas Göttliches gibt? \\
\hline Öffentliche Praxis & Wie häufig nehmen Sie an Gottesdiensten teil? \\
\hline Private Praxis & Wie häufig beten Sie? \\
\hline Erfahrung & $\begin{array}{l}\text { Wie oft erleben Sie Situationen, in denen Sie das Gefühl haben, } \\
\text { dass Gott oder etwas Göttliches in Ihr Leben eingreift? }\end{array}$ \\
\hline & Index der Zentralität \\
\cline { 2 - 2 } &
\end{tabular}

Quelle: Huber 2008b, S. 4

Tab. 2: Indikatoren der Religiosität

Die Indikatoren (Kerndimensionen) werden beim Religionsmonitor 2008 in einen Index der Zentralität der Religiosität eingestellt (vgl. Tab. 2). Mit Zentralität ist hier gemeint, dass die Religion einen umso zentraleren Stellenwert im Leben eines Menschen einnimmt, je höher dessen persönlicher Indexwert ist. Der Summenindex wird gebildet, indem jeder Antwortmöglichkeit ein Zahlenwert von eins bis fünf zugewiesen wird. ${ }^{7)}$ Da die Zentralität der Religiosität aus den fünf Kerndimensionen abgeleitet wird, liegt das Minimum bei fünf und das Maximum bei 25 Punkten. Drei Kategorien der Zentralität werden letztlich erstellt: Nicht-Religiöse, Religiöse und Hoch-Religiöse. Dabei gilt ein Proband als Nicht-Religiöser, wenn sein Indexwert

6) Die Methodik des Religionsmonitors 2008 basiert auf fünf Kerndimensionen der Religiosität: Intellekt, Ideologie, öffentliche Praxis, private Praxis sowie Erfahrung. Alle Dimensionen werden mit einer fünfstufigen Skala erfasst (Huber 2008a, S. 21-28).

7) 1 = nie / gar nicht; $2=$ selten $/$ wenig; 3 = gelegentlich $/$ mittel; 4 = oft $/$ ziemlich; $5=$ sehr oft $/$ sehr 


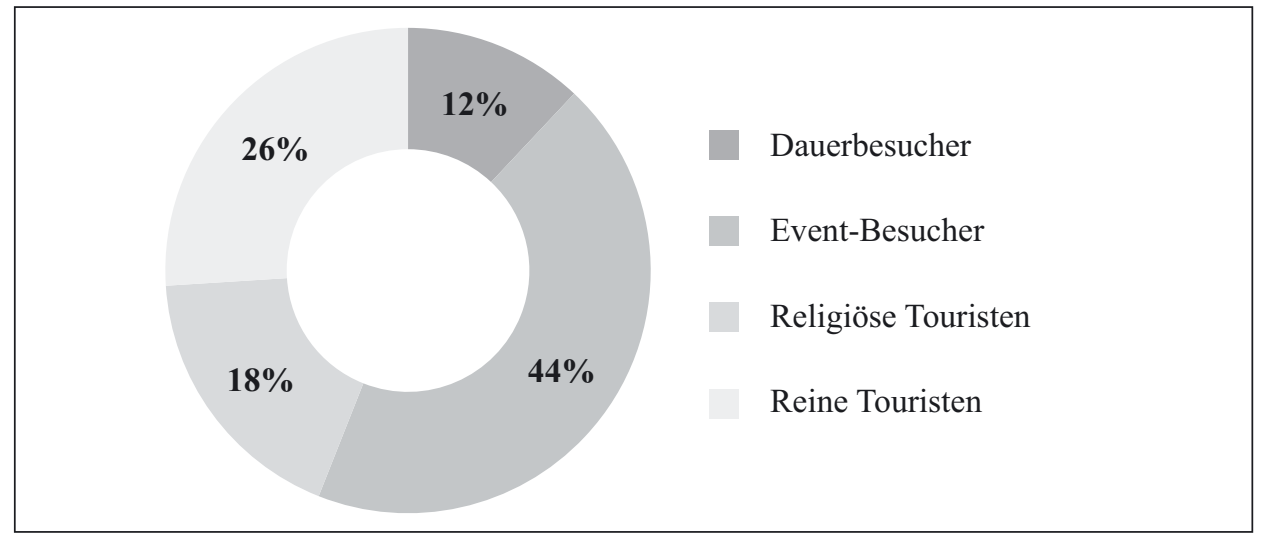

Abb. 6: Besuchertypen

zwischen fünf und einschließlich zehn Punkten liegt. Die Kategorie Religiöse reicht von elf bis einschließlich 19 Punkten. Erreicht ein Befragter einen Wert von 20 oder mehr Punkten, so wird er als Hoch-Religiöser klassifiziert (Huber 2008b, S. 8).

Statistisch lassen sich vier Besucher-Typen an Wallfahrtsorten unterscheiden (vgl. Abb. 6): Die „Dauerbesucher“ (12\%) wallfahren aus spirituellen Gründen regelmäßig und häufig. Zumeist besuchen sie denselben Wallfahrtsort mehrmals im Jahr. Eher selten werden andere Gnadenorte aufgesucht. Der Indexwert der Religiosität weist die Besucher dieses Typs als Hoch-Religiöse aus. Für diese Menschen spielt die Religion eine übergeordnete Rolle in ihrem Leben. Ihre Motive sind in der Volksfrömmigkeit verankert.

Die „Event-Besucher“ (44\%) kommen indes nur zu besonderen Festen und Anlässen an einen Wallfahrtsort. Gründe hierfür sind vor allem der festlichere Rahmen, der an den Gnadenorten geboten wird, oder auch besondere liturgische Angebote, vor allem aus dem Bereich der Marienverehrung. Auch wenn der Begriff des Events nicht zum Bild des andächtigen Wallfahrers passen will, so bestehen doch Berührungspunkte zwischen Volksfrömmigkeit und moderner Festivalisierungskultur. Denn die „Event-Besucher“ an Wallfahrtsorten wollen etwas erleben. Deshalb sucht dieser Typ auch zumeist denselben Wallfahrtsort auf, an dem er etwas geboten bekommt. Hinsichtlich der Religiosität der „Event-Besucher“ bestehen aber lediglich geringe Unterschiede zu den „Dauerbesuchern“. Der „Event-Besucher“ wird ebenfalls von den Hoch-Religiösen dominiert, jedoch lassen sich auch Menschen darunter finden, in deren Leben die Religion eine weniger wichtige Position einnimmt und die somit zu den Religiösen zu zählen sind.

Die „reinen Touristen“ (26\%) sind ausschließlich an Kunst und Kultur des Ortes interessiert und verstehen ihren Besuch lediglich als Mittel der Freizeitgestaltung. Dieser Besuchertyp ist also weit weniger religiös als die beiden erstgenannten. Dass es sich beim besuchten Ort um einen Wallfahrtsort handelt, ist für diesen BesucherTyp nicht relevant. Auch wird derselbe Gnadenort nicht mehrmals aufgesucht, dafür 
werden es aber mehrere verschiedene, auch wenn den Besuchern nicht bewusst ist, dass es sich bei den besuchten Orten um Gnadenstätten handelt.

Im Gegensatz zu den ,reinen Touristen“ verbinden die „religiösen Touristen“ (18\%) ihre Bedürfnisse nach Freizeit und Religion am Wallfahrtsort miteinander. Dies äußert sich in Gottesdienstbesuchen und darin, dass die Wallfahrtsorte auch aufgrund ihrer religiösen Bedeutung als Ausflugsziel ausgewählt wurden und nicht nur wegen ihrer künstlerischen Gestaltung.

Neben der Nachfrage spielt das liturgische Angebot der Wallfahrtsorte bei der Spezialisierung eine zentrale Rolle. Personell besser ausgestattete Wallfahrtsorte bieten in der Regel ein breiteres religiöses Angebot. Aber auch das weltliche Angebot, wie beispielsweise eine besonders wertvolle Ausstattung mit Kunstobjekten oder ein architektonisch besonders interessanter Kirchenbau tragen zur Spezialisierung bei. In gleicher Weise sind die Erreichbarkeit von Gaststätten, die Anbindung an das Verkehrsnetz oder die Möglichkeiten der Freizeitgestaltung in der Umgebung für die Spezialisierung von Bedeutung.

Die Spezialisierung des Angebots wirkt gleichermaßen wieder auf die Sortierung der Nachfrage: So lassen sich Orte finden, die mit einem sehr gut ausgebauten religiösen Angebot vor allem die ersten beiden Besuchertypen ansprechen. Andere ziehen indes durch ihre kunsthistorischen Alleinstellungsmerkmale die touristisch geprägten Besucher, auch aus weiter Ferne, an.

Beispielsweise zieht die Wallfahrtskirche St. Jakobus Major in Biberbach, die das über die bayerisch-schwäbischen Grenzen hinaus bekannte Gnadenbild „Herrgöttle von Biberbach" beherbergt, vor allem Besucher der beiden touristisch orientierten Besucher-Typen an. Dies ist sowohl auf die Lage am Jakobus-Pilgerweg als auch auf das einzigartige Kunstobjekt (Gnadenbild) zurückzuführen. ${ }^{8}$ Hier hat das Vorhandensein eines Kunstobjektes zu einer Spezialisierung im touristischen und nicht im religiösen Bereich geführt.

Ähnlich ist die Besucherzusammensetzung der Wieskirche bei Steingaden. Die Kirche ist aufgrund ihrer barocken Ausgestaltung zum UNESCO-Weltkulturerbe erklärt worden. Daher dominieren auch hier die Besucher-Typen mit touristischen Motiven. Dennoch finden sich auch 19\% Besucher aus den religiös motivierten Typen, weil der Wallfahrtsort zahlreiche liturgische Angebote bereit hält, die neben den zahlreichen Touristen auch andere Zielgruppen ansprechen.

Die Wallfahrtskirchen St. Michael in Violau, Maria Vesperbild und Herrgottsruh in Friedberg bei Augsburg sprechen vor allem „Event-Besucher“ an. Dies ist auf die zahlreichen Angebote aus der Volksfrömmigkeit und die prachtvolle Ausgestaltung religiöser Hochfeste zurückzuführen. Besonders sticht hier Maria Vesperbild heraus.

\footnotetext{
8) Das Biberbacher Kruzifix lässt sich nur äußerst schwierig in eine Epoche einordnen. Es ist zwar der Romanik zuzuordnen, weist jedoch zahlreiche Besonderheiten aus anderen Kunstepochen auf, die den künstlerischen Reiz des Gnadenbildes begründen. So wird Jesus zwar mit allen Wundmalen und einer geflochtenen Seilkrone mit Dornenbesatz, jedoch nicht als toter, sondern lebendiger Christus dargestellt. Auch die Körperhaltung der Christusdarstellung ist als stehend und nicht hängend zu beschreiben. Diese Mischung gegensätzlicher Darstellungsmerkmale verleiht dem Kruzifix aus Biberbach seine künstlerische und kunsthistorische Bedeutung (PöTZL 1997, S. 23ff.).
} 


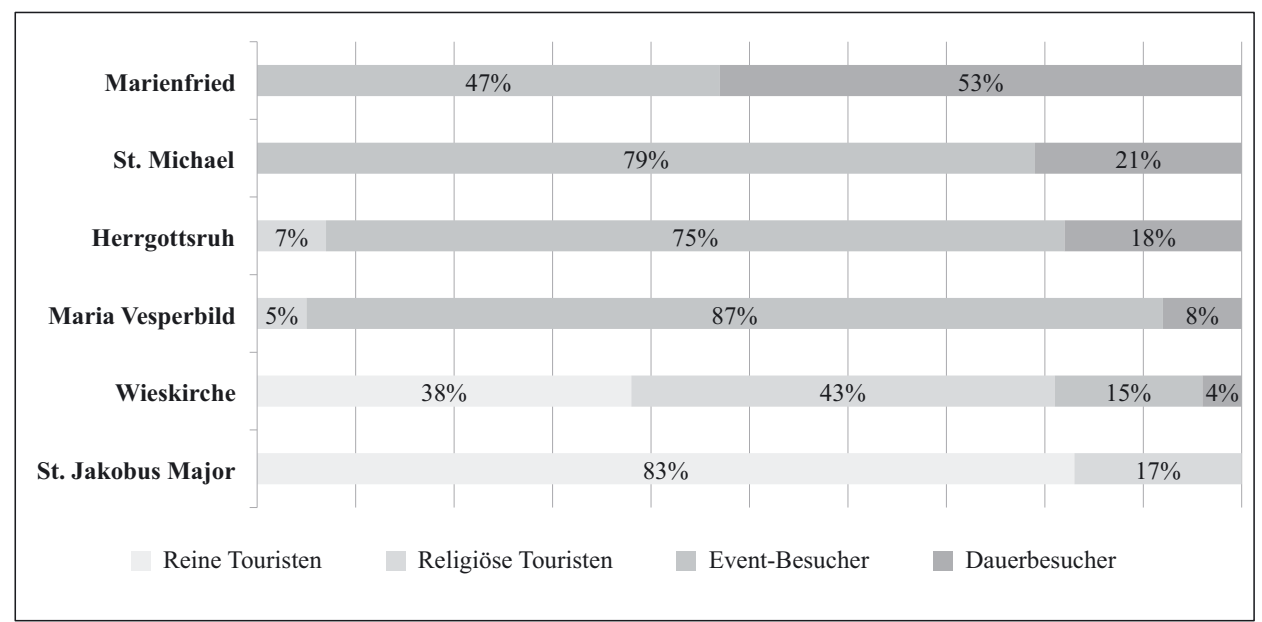

Abb. 7: Besucherzusammensetzung an ausgewählten Wallfahrtsorten

Die Wallfahrtsdirektion bietet im Jahresverlauf zahlreiche religiöse Feiern und Feste an, die über die Grenzen Bayerns hinaus bekannt sind.

Die Gebetsstätte Marienfried hingegen spricht ein anderes Publikum an. Dieser Ort hat sich auf die Marienverehrung spezialisiert und zieht somit ausschließlich die beiden religiös motivierten Besuchertypen besonders an (vgl. Abb. 7). Viele Wallfahrtsorte haben aber aufgrund des säkular bedingten Bedeutungsverlustes keine Spezialisierung erfahren oder diese verloren.

\section{Auswirkungen der Spezialisierung auf das räumliche Muster}

Eine Spezialisierung Zentraler Orte gleicher Hierarchiestufe hat bereits August Lösch nachgewiesen (1944, S. 86-90). Diese Spezialisierung ist die direkte Folge spezifischer Funktionen. So können Orte gleicher Hierarchiestufe unterschiedliche Angebote haben. Auch müssen nicht alle Orte höherer Zentralität zwingend alle Funktionen der Orte niederer Zentralität aufweisen. Die Gnadenorte differenzieren sich somit nicht nur vertikal in Form einer Hierarchie, sondern auch horizontal durch Spezialisierung auf bestimmte Nachfragen aus. Auch ist das räumliche Muster variabler, da teilweise eine Auflösung der Netzwerkstruktur zugunsten überlappender Marktgebiete nötig ist. So entstehen Sektoren mit einer höheren und Sektoren mit einer geringeren Dichte Zentraler Orte.

Diese Erkenntnisse der Theorie der Marktnetze sind von essenzieller Bedeutung für die Erklärung der räumlichen Verteilungsmuster von Wallfahrtsorten. Besonders das Verständnis, dass Orte gleicher Zentralität unterschiedliche Angebote haben können und nicht alle Angebote der niedrigeren Stufen in sich vereinigen, ist bei den Wallfahrtsorten des Bistums Augsburg nachweisbar. Allein schon bei der Untersuchung 
der religiösen Oberzentren lassen sich eindeutige Spezialisierungen feststellen. Als Beispiele dienen die Gebetsstätte Marienfried und die Wallfahrtsdirektion Herrgottsruh in Friedberg. Hier sind vor allem nachfrageseitige (verschiedene Besuchertypen) Unterschiede signifikant: Die Besucher der Gebetsstätte Marienfried sind mehrheitlich „Dauerbesucher“. Der Ort ist gleichsam eine Ausdrucksform tiefster Frömmigkeit, vor allem der Marienverehrung. Dementsprechend organisiert sich auch das Angebot der Gebetsstätte. Es umfasst neben den konventionellen Gottesdiensten beispielsweise auch Sühnenächte und Exerzitien. ${ }^{9)}$ Die Wallfahrtsdirektion Herrgottsruh spricht

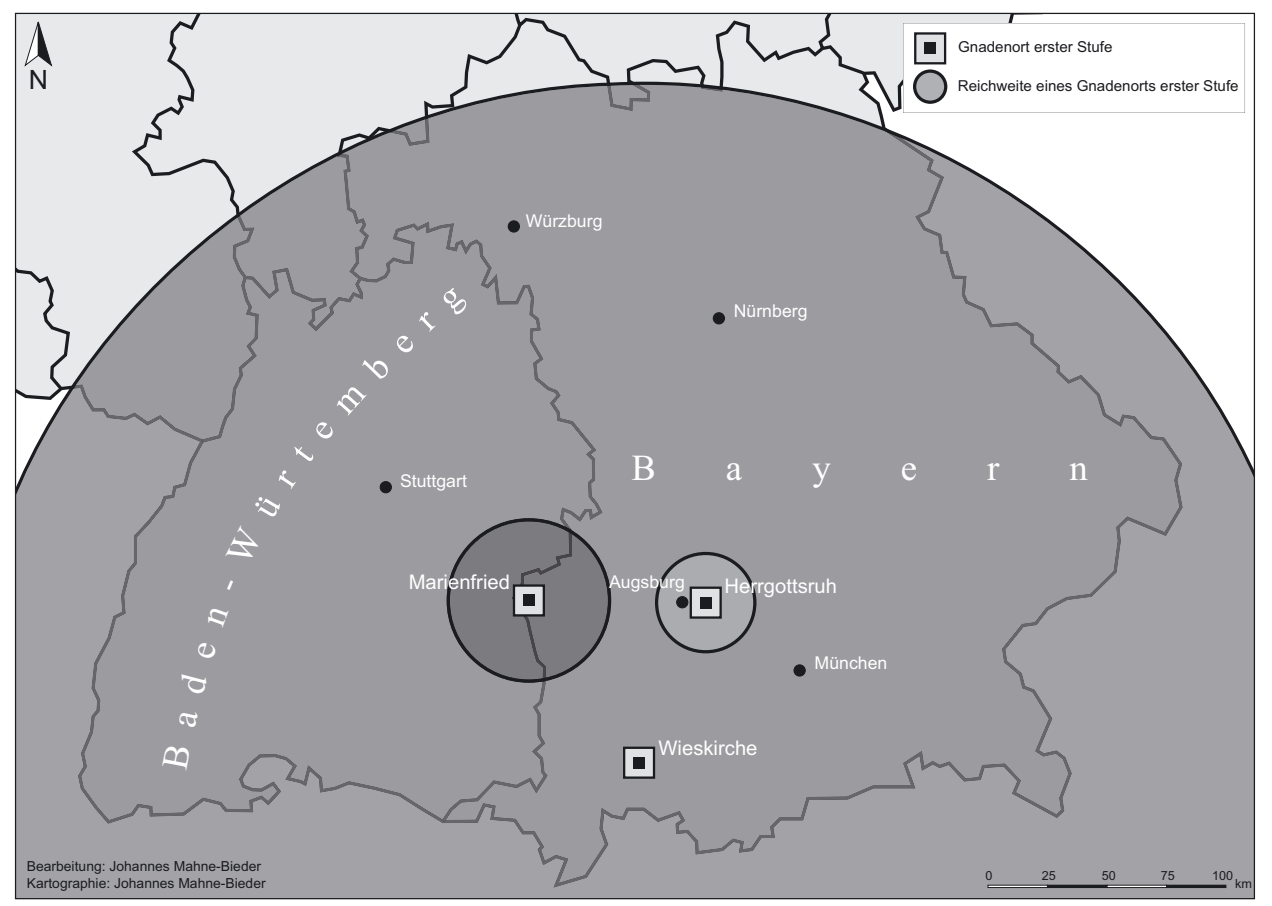

Abb. 8: Einzugsgebiete ausgewählter Wallfahrtsorte ${ }^{10)}$

9) Die Sühnenacht ist eine besondere Art der Andacht, die keiner festgelegten Form unterliegt. Zumeist beinhaltet sie H1. Messen und zahlreiche Gebete. Sie findet, wie der Name schon sagt, über Nacht statt. Exerzitien sind religiöse Übungen, die auf eine Vertiefung des Lebens hin zu Christus zielen. Besondere Bekanntheit erlangten die Exerzitien nach Ignatius von Loyola, der seine Form der geistlichen Übungen 1541 in lateinischer Schrift veröffentlichte. In dieser Form bestehen die Exerzitien aus der Teilnahme an der Eucharistie, der Betrachtung der Evangelien und Phasen der Gewissenserforschung. Die Exerzitien werden zumeist schweigend durchgeführt. Unterschieden werden können heute die großen Exerzitien, welche vier Wochen andauern können, die kleinen Exerzitien mit einer Dauer von etwa einer Woche sowie die mehrwöchigen Exerzitien im Alltag. Eine Sonderform stellen Einkehrtage dar, die aus Besinnung und Gebet bestehen und zumeist nur einige Tage dauern. In jüngerer Zeit hat auch die evangelische Kirche eine ähnliche geistliche Übung entwickelt, die sogenannte Rüstzeit. Diese besteht zumeist aus mehrtägigen Kursen, in denen ein bestimmtes Thema vertieft behandelt wird.

${ }^{10)}$ Die Reichweiten der Wallfahrtsorte wurden mithilfe von Befragungen empirisch ermittelt. Dabei wurde die Postleitzahl des Wohnortes der Probanden erfasst. Zur Ermittlung der maximalen Reichweite wurde der Postleitzahlenbereich mit der größten Entfernung als Radius der dargestellten Reichweiten verwendet. 
andere Besuchergruppen an, nämlich hauptsächlich „Event-Besucher“. Vor allem die zahlreichen Fußwallfahrten, die Herrgottsruh zum Ziel haben, ziehen viele Besucher an, seien es nun Teilnehmer des Wallfahrtszuges oder Zuschauer. Auch das gesellige Beisammensein nach dem gemeinsamen Wallfahrtsgottesdienst ist hier ein zentrales Argument. Das Angebot umfasst folglich auch Feiern, Konzerte und Lesungen.

Beide Orte gehören zu den ,religiösen Oberzentren“, jedoch mit unterschiedlichen Spezialisierungen. Die Gebetsstätte Marienfried wird von Gläubigen aufgesucht, die sich nach einer traditionelleren, zum Teil vorkonsiliarischen Kirche sehnen. Im Gegensatz dazu hat sich die Wallfahrtsdirektion Herrgottsruh mit ihren zahlreichen Bitt- und Dank-Wallfahrten auf die weit verbreiteten Formen der Volksfrömmigkeit spezialisiert.

Die Spezialisierungen der Wallfahrtsorte haben darüber hinaus auch räumliche Konsequenzen, vor allem für die Einzugsgebiete der Gnadenstätten mit einer stark ausgeprägten Spezialisierung in einer kleinen Marktnische. So bietet im Bistum Augsburg neben Marienfried lediglich die Gebetsstätte Wigratzbad ein ähnliches Angebot. Diese Nischenposition hat zur Folge, dass die Einzugsgebiete der beiden Gebetsstätten überproportional groß ausfallen. Ähnlich verhält es sich beim Kloster Andechs (bzw. der dazugehörigen Brauereiwirtschaft) oder bei der Wieskirche in Steingaden, die als UNESCO-Weltkulturerbe wohl zu den kunsthistorisch bedeutsamsten Kirchen Deutschlands oder gar Europas zählt (vgl. Abb. 8).

\section{Schluss}

Um sicher eine vollständige Übertragbarkeit auf größere Räume, wie etwa Bayern, Deutschland oder gar Europa annehmen zu können, genügen die Ergebnisse der beschriebenen Studie nicht. Vor allem die Spaltung der christlichen Kirche erschwert in Deutschland die Übertragbarkeit auf vornehmlich evangelische Gebiete, wo zahlreiche Wallfahrtsorte heute nicht mehr existieren. Des Weiteren ist unklar, ob die hier als „religiöse Oberzentren“ identifizierten Gnadenstätten auch in einem gesamtdeutschen oder europäischen Kontext die höchste Hierarchiestufe besetzten. Dennoch konnte nachgewiesen werden, dass zumindest im Bistum Augsburg Wallfahrtsorte derselben Hierarchiestufe unterschiedliche Spezialisierung mit einem entsprechenden Angebot erfuhren. Daraus resultieren räumliche Konsequenzen, wenngleich die Wallfahrtsorte, verstanden als Orte religiöser Dienstleistungen, prinzipiellen Gesetzmäßigkeiten Zentraler Orte folgen.

\section{Literaturverzeichnis}

Bistum Augsburg (2011), Liste der Wallfahrtsorte im Bistum Augsburg. Augsburg.

BRÜCKNER W. (2000), Kulturtechniken. Nonverbale Kommunikation, Rechtssymbolik, religio carnalis (= Veröffentlichungen zur Volkskunde und Kulturgeschichte, IX). Bamberg, Lehrstuhl für Europäische Ethnologie. 
Christaller W. (1933), Die zentralen Orte in Süddeutschland. Eine ökonomisch-geographische Untersuchung über die Gesetzmäßigkeit der Verbreitung und Entwicklung der Siedlungen mit städtischen Funktionen. Jena, Fischer.

Höllhuber D., Kaul W. (1987), Wallfahrt und Volksfrömmigkeit in Bayern. Nürnberg, Carl. Huber S. $\left(2008 \mathrm{a}^{2}\right)$, Aufbau und strukturierende Prinzipien des Religionsmonitors. In: BertelsMAnN-Stiftung (Hrsg.), Religionsmonitor, S. 19-29. Gütersloh, Bertelsmann-Stiftung.

Huber S. (2008b), Religionsmonitor. Kurzbericht zu ersten Ergebnissen des Religionsmonitors der Bertelsmann-Stiftung. - http://www.bertelsmann-stiftung.de/bst/de/media/ $x c m s \_b s t d d m s \_23407 \_23408 \_2 . p d f($ Zugriff am 21.1.2010).

Ioannes Paulus PP. II, Aymans W. (Hrsg.) (20015), Codex des kanonischen Rechts. Kevelaer, Butzon \& Bercker.

Kriss R. (1956), Die Volkskunde der altbayerischen Gnadenstätten, Band III: Theorie des Wallfahrtswesens. München, Filser.

Kriss R. (1963), Zur Begriffsbestimmung des Ausdruckes „Wallfahrt“. In: Österreichische Zeitschrift für Volkskunde, 66, S. 101-107.

Lösch A. (1944²), Die räumliche Ordnung der Wirtschaft. Jena, Fischer.

Mahne-Bieder J. (2010), Die Wallfahrtsorte und Gebetsstätten des Bistums Augsburg und ihre Besucher. Unveröffentlichtes Manuskript. Augsburg.

Pötzl W. (1997), Spuren romanischer Kunst. In: PötzL W. (Hrsg.), Der Landkreis Augsburg, Band 6: Kunstgeschichte, S. 10-28. Augsburg, Landratsamt Augsburg.

Renkikowski C. (2009), Wallfahrt: Die älteste Form des Massentourismus. - http://www.katho lisch.de/Nachricht.aspx?NId=1852 (Zugriff am 06.06.2010).

Rieger M. (2007), Einleitung. In: Bertelsmann-Stiftung (Hrsg.), Religionsmonitor 2008, S. 11-17. Gütersloh, Bertelsmann-Stiftung.

Rinschede G. (1985), Das Pilgerzentrum Lourdes. In: Büttner M. (Hrsg.), Grundfragen der Religionsgeographie, S. 195-256. Berlin, Reimer.

RinschedE G. (1999), Religionsgeographie. Braunschweig, Westermann. 